\title{
Correction to: Adverse events following immunization with pentavalent vaccine: experiences of newly introduced vaccine in
} Iran

Manoochehr Karami ${ }^{1,2^{*}}$, Pegah Ameri ${ }^{2}$, Jalal Bathaei ${ }^{3}$, Zeinab Berangi ${ }^{2}$, Tahereh Pashaei ${ }^{4}$, Ali Zahiri ${ }^{3}$, Seyed Mohsen Zahraei ${ }^{5}$, Hussein Erfani ${ }^{3}$ and Koen Ponnet ${ }^{6,7}$

\section{Erratum}

After publication of the original article [1], the authors noticed that the percentages in Table 2 were incorrect.

The correct version of Table 2 is published in this erratum.

\begin{abstract}
Author details
${ }^{1}$ Social Determinants of Health Research Center, Hamadan University of Medical Sciences, Hamadan, Iran. ${ }^{2}$ Department of Epidemiology, School of Public Health, Hamadan University of Medical Sciences, Hamadan, Iran. ${ }^{3}$ Deputy for Health, Hamadan University of Medical Sciences, Hamadan, Iran. ${ }^{4}$ Social Determinants of Health Research Center, Kurdistan University of Medical Sciences, Sanandaj, Iran. ${ }^{5}$ Center for Communicable Diseases Control, Ministry of Health, Tehran, Iran. ${ }^{6}$ Department of Communication Studies, Ghent University, Ghent, Belgium. ${ }^{7}$ Faculty of Social Sciences, University of Antwerp, Antwerp, Belgium.
\end{abstract}

Received: 20 September 2017 Accepted: 21 September 2017

Published online: 05 October 2017

\section{Reference}

1. Karami $\mathrm{M}$, et al. Adverse events following immunization with pentavalent vaccine: experiences of newly introduced vaccine in Iran. BMC Immunol. 2017:18:42. doi:10.1186/s12865-017-0226-8.

\footnotetext{
* Correspondence: ma.karami@umsha.ac.ir

'Social Determinants of Health Research Center, Hamadan University of Medical Sciences, Hamadan, Iran

${ }^{2}$ Department of Epidemiology, School of Public Health, Hamadan University

of Medical Sciences, Hamadan, Iran
} 
Table 2 Comparison of the cumulative incidence rates of complications in the first, second and third doses of the vaccination in children

\begin{tabular}{|c|c|c|c|c|c|}
\hline \multirow[t]{2}{*}{ Adverse events } & \multicolumn{3}{|c|}{$\begin{array}{l}\text { Incidence (per } 100 \text { children) } \\
\text { Vaccine dose }\end{array}$} & \multirow{2}{*}{$\begin{array}{l}P \text {-value } \\
\left(1^{\text {st }} \text { and } 2^{\text {nd }} \text { dose }\right. \\
\text { comparison) }\end{array}$} & \multirow{2}{*}{$\begin{array}{l}P \text {-value } \\
\left(2^{\text {nd }} \text { and } 3^{\text {rd }} \text { dose }\right. \\
\text { comparison) }\end{array}$} \\
\hline & $\begin{array}{l}1^{\text {st }} \text { dose } \\
(2 \text { months }) \\
N=373\end{array}$ & $\begin{array}{l}2^{\text {nd }} \text { dose } \\
\text { (4 months) } \\
N=372\end{array}$ & $\begin{array}{l}3^{\text {rd dose }} \\
\text { (6 months) } \\
N=374\end{array}$ & & \\
\hline Swelling & $79(21.2)$ & $50(13.4)$ & $48(12.8)$ & 0.003 & 0.068 \\
\hline Redness & $50(13.4)$ & $30(8.1)$ & $43(11.5)$ & 0.026 & 0.679 \\
\hline Pain & 190(50.9) & 143(38.4) & $62(16.6)$ & 0.001 & 0.162 \\
\hline Mild fever & $45(12.1)$ & $33(8.9)$ & $63(16.8)$ & 0.068 & $<0.001$ \\
\hline High fever & $1(0.3)$ & $0(0)$ & $0(0)$ & 0.388 & NA \\
\hline Drowsiness & $83(22.3)$ & 72(19.4) & $70(18.7)$ & 0.310 & 0.725 \\
\hline Anorexia & $65(17.4)$ & $51(13.7)$ & $52(13.9)$ & 0.126 & 1 \\
\hline Restlessness & $35(9.4)$ & 115(30.9) & 119(31.8) & $<0.001$ & 0.766 \\
\hline Vomiting & $16(4.3)$ & $19(5.1)$ & $17(4.5)$ & 0.510 & 0.510 \\
\hline Long-term crying & $24(6.4)$ & $16(4.3)$ & $22(5.9)$ & 0.210 & 0.510 \\
\hline Encephalopathy & $0(0)$ & $0(0)$ & $0(0)$ & NA & NA \\
\hline Convulsion & $0(0)$ & $0(0)$ & $0(0)$ & NA & NA \\
\hline History of convulsion & $0(0)$ & $1(0.3)$ & $2(0.5)$ & 0.387 & 0.488 \\
\hline Family history of convulsion & $0(0)$ & 14(3.8) & $6(1.6)$ & $<0.001$ & 0.074 \\
\hline
\end{tabular}

NA not applicable 